\title{
Voltage Quality Factor for Networks Supplying Unbalanced Nonlinear Loads
}

\author{
Juan-Carlos Montaño ${ }^{1}$, Dolores Borrás ${ }^{2}$ Manuel Castilla ${ }^{2}$, Antonio López ${ }^{3}$, Jaime Gutiérrez ${ }^{4}$ and Juan-Carlos \\ Bravo $^{2}$ \\ ${ }^{1}$ Spanish Research Council (CSIC), IRNAS, Reina Mercedes Campus, \\ P. O. Box 1052, 41080 Sevilla, (Spain) \\ phone:+34.9546 24711, fax:+34 9546 24002, e-mail: montano@irnase.csic.es \\ ${ }^{2}$ Electrical Engineering Department. University of Sevilla, \\ Escuela Universitaria Politécnica, Virgen de Africa, 7, 41011-Sevilla (Spain) \\ e-mail: borras@platero.eup.us.es, castilla@us.es, carlos_bravo@us.es \\ ${ }^{3}$ Electronic Technology Department. University of Sevilla, Spain \\ e-mail: alojeda@us.es \\ ${ }^{4}$ Applied Physics Department. University of Sevilla, Spain \\ e-mail: jaiben@us.es
}

\begin{abstract}
While the common term for describing the harmonic content and the unbalanced loading of the three-phase supply is power quality, it is actually the quality of the voltage that is being addressed in most cases. The power supply system can only control the quality of the voltage; it has no control over the currents that particular loads might draw. Therefore, the standards in the power quality area are devoted to maintaining the voltage within certain limits.

In this paper, a voltage quality factor, designated $\mathrm{VQF}$, is suggested that integrally assesses the voltage quality of a given three phase supply from the two aspects mentioned above.
\end{abstract}

\section{Key words}

Power quality, harmonic distortion, three-phase systems, nonlinear circuits, power systems.

\section{Introduction}

The harmful and costly effects of harmonics have been discussed extensively [1], [2] and spurred stringent requirements by international institutions regarding the allowed levels of harmonics at the point of connection to the power supply. Unbalanced loading of the three-phase supply has no less detrimental effects such as the under utilization of the power supply equipment and overloading of neutral conductors with fundamental frequency in addition to harmonic currents.

While the common term for describing these subjects is power quality [3], it is actually the quality of the voltage that is being addressed in most cases. The power supply system can only control the quality of the voltage; it has no control over the currents that particular loads might draw. Therefore, the standards in the power quality area are devoted to maintaining the voltage within certain limits.
In this paper, a voltage quality factor, designated $V Q F$, is suggested that integrally assesses the voltage quality of a given three phase supply from the two aspects mentioned above.

\section{Quantitative Formulations of Voltage Quality Aspects}

\section{A. Total Voltage Harmonic Distortion}

The total harmonic distortion of the voltage VTHD for single phase (or polyphase balanced networks) has been conventionally defined [4-6] in the literature as

$$
V T H D=\frac{\sqrt{\sum_{h \neq 1} V_{h}^{2}}}{V_{1}}
$$

where $V$ denotes rms value and 1 and $h$ denote the fundamental and the harmonic order respectively.

An extension of the above concepts to unbalanced polyphase networks has been suggested. To this end a single 'equivalent' harmonic rms voltage $V_{e H}$ for the three phase system $a, b, c$ is defined [7] as

$$
V_{e H}=\sqrt{\sum_{h \neq 1} \frac{V_{a h}^{2}+V_{b h}^{2}+V_{c h}^{2}}{3}}
$$

The first quality aspects $Q A_{1}$ is identified with the total harmonic distortions $V T H D$ for a three-phase unbalanced system and is given by

$$
Q A_{l}=V T H D=\frac{V_{e H}}{V_{e 1}}
$$

where $V_{e l}$ denotes the fundamental equivalent phase voltage defined, similarly to (2), from the fundamental components of the generally unbalanced three-phase 
voltages as

$$
V_{e 1}=\sqrt{\frac{V_{a 1}^{2}+V_{b 1}^{2}+V_{c 1}^{2}}{3}}
$$

\section{A. Degree of Unbalance}

As is well known, unbalanced voltages in polyphase networks affect the quality of voltage in many aspects, such as increased line losses for the same power transfer level, extra rotating losses in drives and overloading of neutral conductors in four-wire distribution systems.

Applying the theory of symmetrical components [8], an unbalanced three-phase sinusoidal voltage system $\left[V_{a}, V_{b}\right.$, $V_{c}$ ] can be decomposed into a positive sequence threephase balanced system $V^{+}$, a negative sequence system $V$ and a zero sequence system $V^{0}$ according to

$$
\begin{aligned}
& \underline{V}^{+}=\frac{1}{\sqrt{3}}\left(\underline{V}_{a}+a \underline{V}_{b}+a^{2} \underline{V}_{c}\right) \\
& \underline{V}^{-}=\frac{1}{\sqrt{3}}\left(\underline{V}_{a}+a^{2} \underline{V}_{b}+a \underline{V}_{c}\right) \\
& \underline{V}^{0}=\frac{1}{\sqrt{3}}\left(\underline{V}_{a}+\underline{V}_{b}+\underline{V}_{c}\right)
\end{aligned}
$$

where $V$ denotes the phasor of $V$ and the factor $a=$ $\exp (j 2 \pi / 3)$. After some manipulations of the above equations, the equivalent voltage can be expressed as

$$
V_{e 1}=\sqrt{\underline{V}^{+2}+\underline{V}^{-2}+\underline{V}^{02}}
$$

Pertinent quality aspect $Q A_{2}$ is the voltage unbalance factor $V U N B$ defined as

$$
Q A_{2}=V U N B=\frac{\sqrt{V_{e 1}^{2}-\left(V^{+}\right)^{2}}}{V_{e 1}}
$$

For balanced three-phase voltages it can readily be shown from (5) that $\underline{V}$, and $\underline{V}^{0}$, are equal to zero, $V_{e l}=V^{+}$, and hence $Q A_{2}=0$.

\section{The Voltage Quality Factor}

\section{A. Definition and advantages.}

A single measurable indicator, designated Voltage Quality Factor $(V Q F)$, is suggested to integrally reflect the two voltage quality aspects formulated in the last section. This is expressed as

$$
V Q F=\sum_{i} w_{i}\left(1-Q A_{i}\right)
$$

where $w_{i}$ are judiciously selected weighting factors that sum up to one and $Q A_{i}$ are the different quality aspects formulated in section 2 .

A balanced loaded network, with sinusoidal voltages yield an ideal $V Q F$ of unity. Conversely, a low value of $V Q F$ would indicate a high level of harmonics and/or a high degree of unbalance between the phases with the contribution of each aspect well defined and measurable as illustrated later.

The weighting factors $w_{i}$ together with the recommended value of $V Q F$ are optimally selected so as to reflect the economic and technical importance of a high voltage quality in each of its aspects.

Notable features of definition (8) are

a) it can be expanded to include additional voltage quality aspects ( $Q A$ 's) such as voltage sags and swells adequately defined in terms of their frequency and/or amplitudes, or current quality aspects considering currents instead of voltages.

b) the weighting factors are selected to reflect technical priorities and objectives with due consideration to economic and practical issues. These factors may (and should) be different in different situations and environments.

\section{B. Measurement of $V Q F$}

A conceptual block diagram of a device that measures the $V Q F$ as defined in (8) at a chosen location in a power system is shown in Fig. 1. The device offers the additional feature of distinctly measuring the different quality aspects formulated in sec. 2 so that, if necessary, the voltage transfer aspect that needs correction is identified. The direct measurement of $V Q F$ offers the convenience of assessing the voltage quality by means of a single indicator rather than requiring separate measurements of various entities and individually comparing them with their respective recommended values.

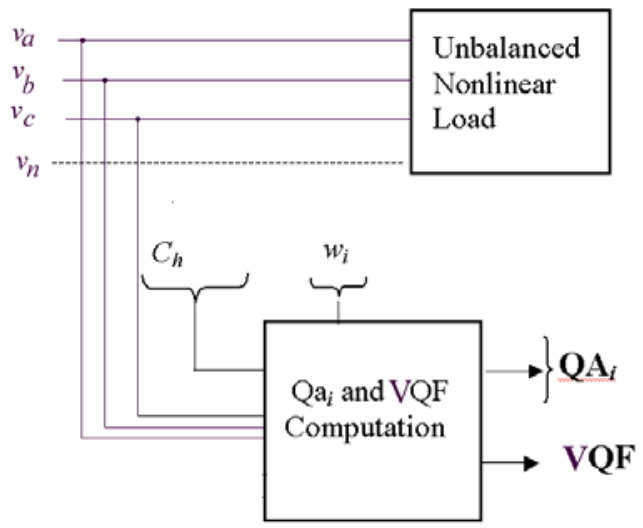

Fig. 1 Conceptual block diagram for the measurement of the Voltage Quality Factor and assessing of the different quality aspects in a three phase supply.

\section{The VQF Virtual Meter}

Voltage waveforms derived from the electric line were simultaneously sampled, converted and processed for monitoring the voltage quality factor and its components. It corresponds to the realization of a virtual equipment which permits to obtain the rms value of the first 63 harmonics and the DC component of the three-phase 


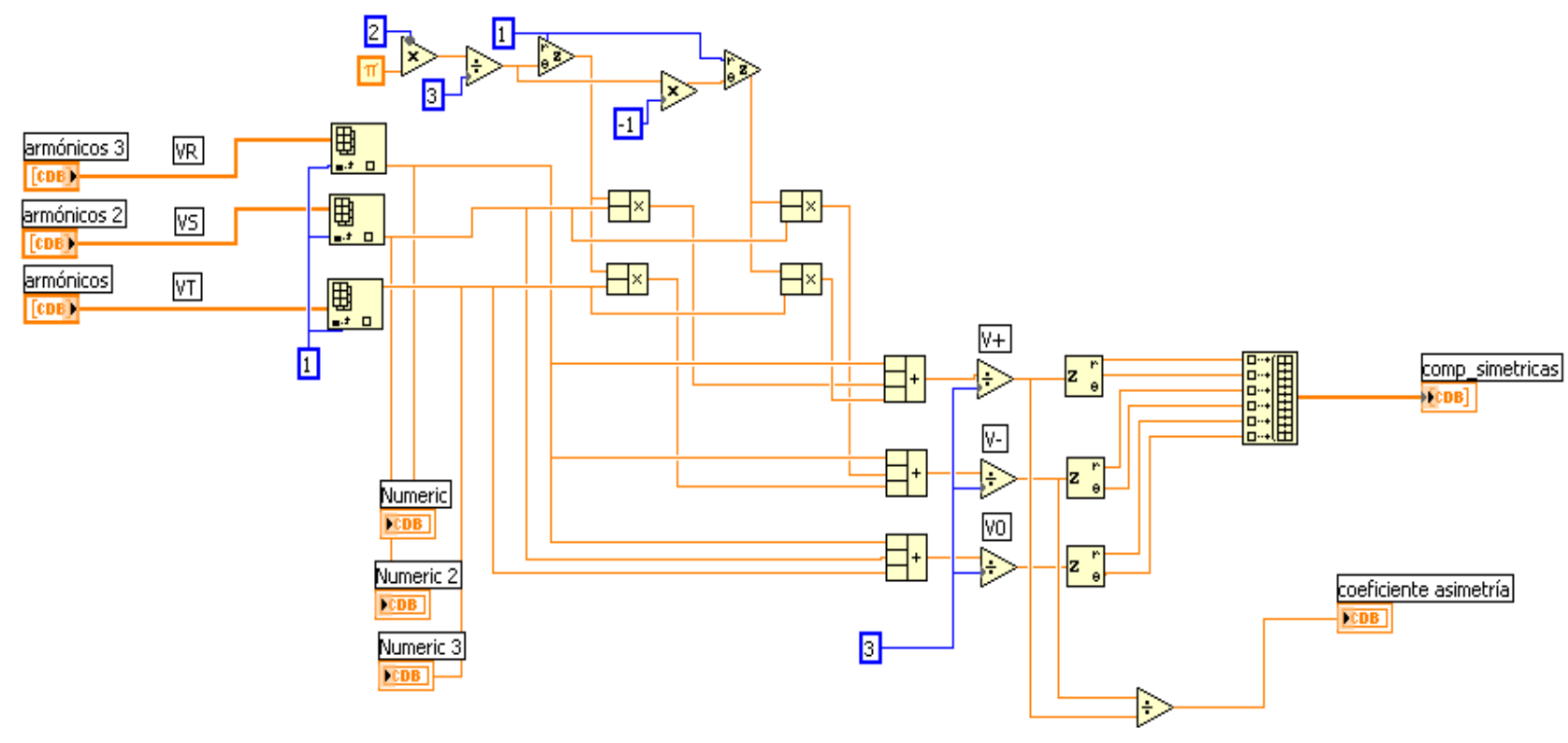

Fig. 2 LabView program corresponding to the Unbalance Factor calculation

voltage signals. All these data are necessary for obtaining the electric quantities:

- RMS values of the voltage signals $\left(V_{a}, V_{b}, V_{c}\right)$ corresponding equivalent value: $V_{e}$.

- Symmetrical components of the three-phase fundamental voltage $\left(V^{+}, V, V^{0}\right)$ components.

- Total Harmonic Distortion Factor of phase voltages VTHD.

- Unbalance Factor of phase voltages $V U N B$.

- $\quad$ The voltage quality factor $V Q F$.

Prior to these calculations the instantaneous- frequency of the voltage signal is obtained, which is necessary to avoid errors due to lack of synchronization between the signal period and the sampling sequence. Thus corrected samples of the set of input signals are obtained for further digital processing based on the FFT. The relative uncertainties that affect the determination of the frequency-domain components and equivalent quantities have been determined experimentally. The uncertainty in the measurement of the amplitude of the frequencydomain components was found to be less than $0.2 \%$ at the fundamental frequency and less than $3.5 \%$ at the higher harmonic frequency. The relative uncertainties in the measurement of the equivalent quantities were found to be less than $1 \%$.

\section{A) Hardware}

A three-phase analyzer was constructed to operate on line, with three channels of phase voltages. The VQM is a device that consists of three voltage transducers, a data acquisition board (PCI-MIO 16E-4 of National Instruments) adapted to a personal computer and a program of control, installed in the PC. Voltage sensors are built with Hall effect voltage transducers, type LV25P. The data acquisition board can acquire eight differential inputs using CMOS analog input multiplexers with overvoltage protection. Analog inputs are converted with 12 bits of resolution, sampled at $1.25 \mathrm{MS} / \mathrm{s}$. The three-phase analyzer is connected to the mains to measure the three-phase quantities corresponding to quality aspects $Q A_{i}$. All the measures are made cycle to cycle. The instantaneous frequency is calculated with a precision of the $0,02 \%$, within the margin of 40 to 60 Hertz. All the three-phase quantities and general functions (collection of information, disk storing, etc.) are controlled by the PC.

\section{Software}

A graphical programming language (LabVIEW 5.0) is used to create the user interface that gives interactive control of the software system. LabVIEW is integrated with the data adquisition board for data development, analysis and presentation solutions.

The measurement results are stored in different files according to the type of information recorded. They are shown in the captured screen of figure 2:

- Instantaneous voltage values of input signals.

- Electrical quantities $Q A i$ of voltage signals defined for the three-phase system.

- Weighting factors $w_{i}$.

- Voltage Quality Factor.

The above data was recorded at $13.30 \mathrm{pm}$ in electrical mains of the second floor of the IRNAS building.

\section{Conclusion}

A general integral assessment of the voltage quality of a three phase network by means of a new indicator designated the voltage quality factor $(V Q F)$ is suggested. The $V Q F$ considers two quality aspects ( $Q A$ 's) the voltage harmonic levels and the degree of unbalance in the different phase voltages. The VQF offers the convenience of assessing the voltage quality by means of 
a single indicator. Moreover, by selecting appropriate weightings, the new voltage factor can be adapted to different economic objectives and load environments.

\section{Acknowledgement}

This work was supported by the 'Comisión Interministerial de Ciencia y Tecnología' (CICYT) of the Spanish Minister of Education and Culture.

\section{References}

[1] V. E. Wagner, J.C. Balda, T.M. Barnes, A.E. Emannuel, R.J. Ferraro, D.C. Griffith, D.P. Hartmann, W.F. Horton, W.T. Jewell, A. McEachern, D.J. Phileggi, and W.E. Reid, "Effects of harmonics on equipment," IEEE Trans. Power Delivery, vol. 8, pp. 672-680, April 1993.

[2] R. D. Henderson and P. J. Rose, "Harmonics: the effects on power quality and transformers," IEEE Trans. Ind. Applicat., vol. 30, pp. 528-532, May/June 1994.

[3] Recommended Practices and Requirements for Harmonic Control in Power Systems, IEEE Standard 519-92.

[4] Limits for Harmonic Current Emissions, IEC 61000, Part 3, Section 4, 1995.

[5] G.T. Heydt, "Electric Power Quality", Stars in a Circle Publication, Lafayette, Indiana, 1991.

[6] A. Mc Eachern, W.M. Grady, W.A. Moncrief, G.T. Heydt and M. McGranaghan,"Revenue and Harmonics: An evaluation of some proposed rate structures," IEEE Trans. Power Delivery, vol. 10, pp. 474-482, Jan. 1995.

[7] IEEE Working Group on nonsinusoidal situations, "Practical definitions for powers in systems with nonsinusoidal waveforms: A discussion," IEEE Trans. Power Delivery, vol. 11, pp. 79-101, January 1996.

[8] C. L. Fortescue, "Method of symmetrical coordinates applied to the solution of polyphase networks," Trans. AIEE, pt. II, vol. 37 pp.1027-1140, 1918.

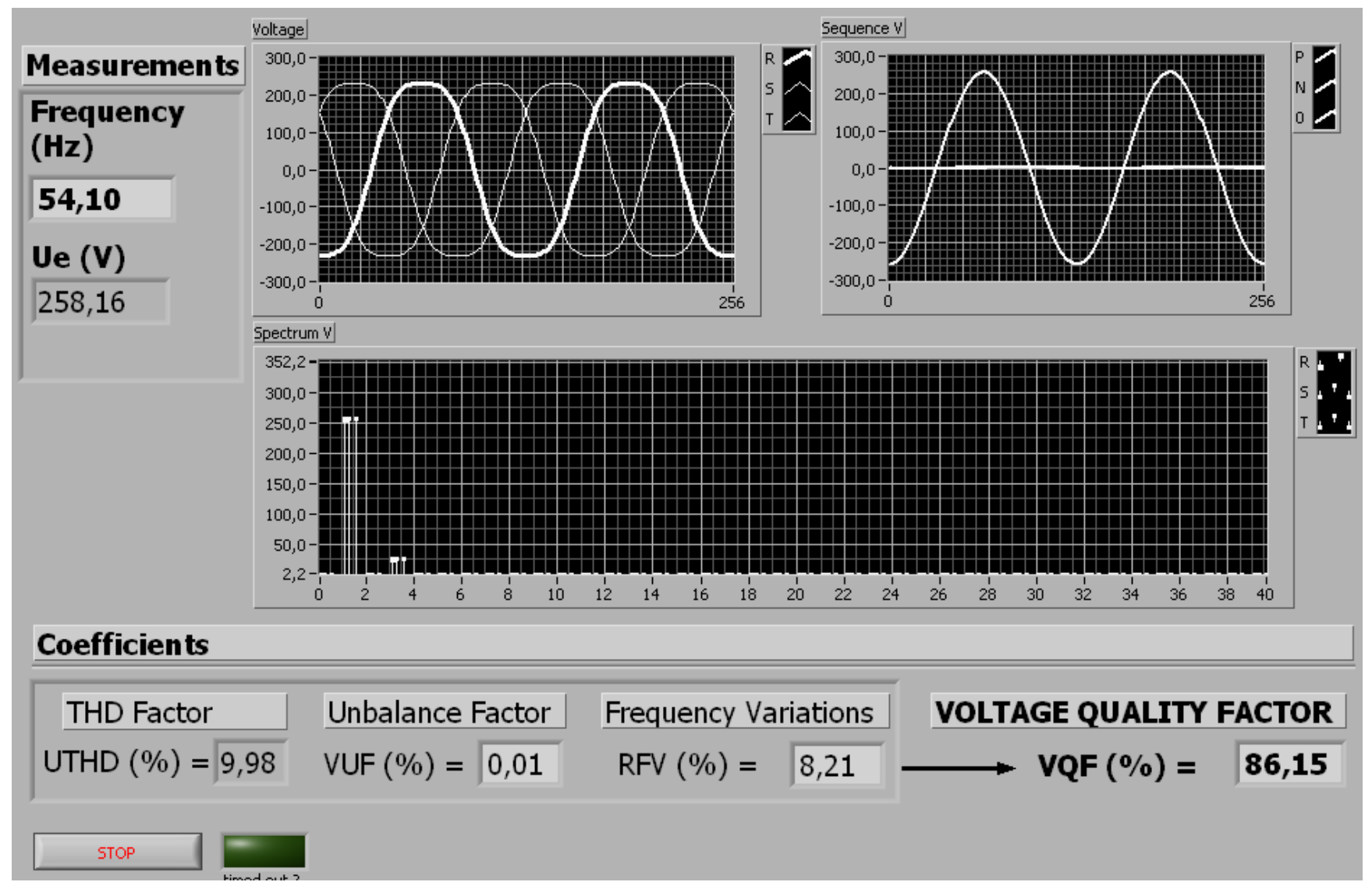

Fig. 3. Screen of the Experimental Voltage Quality Meter. 